\title{
A Case of Refractory Hypoxemic Respiratory Failure due to Antineutrophil Cytoplasmic Antibodies-associated Diffuse Alveolar Hemorrhage Rescued by Extracorporeal Membrane Oxygenation
}

\author{
Manoj K Goel ${ }^{1}$, Munish Chauhan ${ }^{2}$, Ajay Kumar ${ }^{3}$, Pooja Wadwa ${ }^{4}$, Gargi Maitra ${ }^{5}$, Milind Talegaonkkar ${ }^{6}$, Sandeep Dewan ${ }^{7}$
}

\begin{abstract}
Diffuse alveolar hemorrhage (DAH) is a rare but life-threatening disease. Mortality is very high in those patients who require mechanical ventilation. Traditionally, active bleeding has been considered a contraindication for extracorporeal membrane oxygenation (ECMO) support. There is limited evidence for ECMO in DAH as rescue therapy. Herein, we describe a case of antineutrophil cytoplasmic antibodies-associated DAH with intractable hypoxemic respiratory failure. An appropriate ventilator strategy failed to improve her hypoxemia leading to imminent risk to her life. The patient was rescued with veno-venous ECMO targeting lower than usual range of anticoagulation. ECMO proved to be lifesaving in our patient who was initiated on prompt immunosuppressive therapy and plasmapheresis along with continuous veno-venous hemodiafiltration and hemodynamic support. We feel that ECMO could be considered as adjunctive therapy in severe hypoxemic respiratory failure associated with DAH after careful consideration of the risk of bleeding and a restrictive anticoagulation strategy.

Keywords: Antineutrophil cytoplasmic antibodies-associated vasculitis, Diffuse alveolar hemorrhage, Extracorporeal membrane oxygenation. Indian Journal of Critical Care Medicine (2020): 10.5005/jp-journals-10071-23585
\end{abstract}

\section{INTRODUCTION}

Diffuse alveolar hemorrhage (DAH) is a feature of several immune and nonimmune disorders characterized by generalized bleeding into the acini of the lung parenchyma presenting with the cardinal clinical syndrome of hemoptysis, anemia, diffuse radiographic pulmonary infiltrates, and hypoxemic respiratory failure. Antineutrophil cytoplasmic antibodies (ANCA)associated vasculitis (AAV) is the most common cause of DAH. Immunosuppressive therapy is the first-line treatment for AAVrelated $\mathrm{DAH}$, but the management of catastrophic respiratory failure may become a priority in severe cases that are refractory to conventional mechanical ventilation. The Extracorporeal Life Support Organization (ELSO) general guidelines recommend that extracorporeal membrane oxygenation (ECMO) should be considered when the expected mortality is higher than $50 \%$ despite optimized ventilatory care. ${ }^{1}$ There is limited evidence for ECMO in DAH as a rescue therapy that can support gas exchange, providing the time necessary for definitive treatment, especially the immunosuppressive medications to take control of the underlying disease. ${ }^{2,3}$ Herein, we present a case of DAH due to AAV with lifethreatening respiratory failure managed by ECMO. The literature search revealed that only one such case has been reported earlier from India. ${ }^{2}$

\section{Case Description}

A 34-year-old non-smoker, nonalcoholic female presented with complaints of cough and hemoptysis for 2 days and breathing difficulty for 1 day. There was no history of any comorbid illness. On general physical examination she had rapid shallow breathing with a respiratory rate of $40 /$ minute, pulse rate 146 beats/minute, and blood pressure $80 / 60 \mathrm{~mm} \mathrm{Hg}$. On room air, her oxygen
1,3,5 Department of Pulmonology, Critical Care and Sleep Medicine, Fortis Memorial Research Institute, Gurugram, Haryana, India

2,4,6,7 Department of Critical Care Medicine, Fortis Memorial Research Institute, Gurugram, Haryana, India

Corresponding Author: Manoj K Goel, Department of Pulmonology, Critical Care and Sleep Medicine, Fortis Memorial Research Institute, Gurugram, Haryana, India, Phone: +91 9560468800, e-mail: drgoelmanoj@yahoo.co.in

How to cite this article: Goel MK, Chauhan M, Kumar A, Wadwa P, Maitra G, Talegaonkkar M, et al. A Case of Refractory Hypoxemic Respiratory Failure due to Antineutrophil Cytoplasmic Antibodiesassociated Diffuse Alveolar Hemorrhage Rescued by Extracorporeal Membrane Oxygenation. Indian J Crit Care Med 2020;24(9): 879-881.

Source of support: Nil

Conflict of interest: None

saturation $\left(\mathrm{SpO}_{2}\right)$ was $37 \%$. The examination of the respiratory system demonstrated bilateral fine crepts. The arterial blood gas (ABG) findings showed $\mathrm{pH} 7.04, \mathrm{pO}_{2} 41.5 \mathrm{~mm} \mathrm{Hg}, \mathrm{pCO}_{2} 77.9 \mathrm{~mm} \mathrm{Hg}$, and bicarbonate $16 \mathrm{mmol} / \mathrm{L}$, indicating severe respiratory acidosis, hypoxia, and hypercapnia. The patient was immediately intubated and mechanically ventilated. The initial ventilator settings were controlled mechanical ventilation (CMV) mode with tidal volume (TV) of $300 \mathrm{~mL}$, respiratory rate (RR) 32/minute, positive endexpiratory pressure (PEEP) titrated up to $10 \mathrm{~cm} \mathrm{H}_{2} \mathrm{O}$, and fraction of inhaled oxygen $\left(\mathrm{FiO}_{2}\right) 100 \%$. She was hemodynamically supported with intravenous fluid resuscitation and noradrenaline infusion. Her diagnostic workup showed $\mathrm{Hb} 6.2 \mathrm{~g} \%$, ESR $85 \mathrm{~mm} /$ hour, serum urea $78 \mathrm{mg} / \mathrm{dL}$, serum creatinine $4 \mathrm{mg} / \mathrm{dL}$, and C-reactive protein 
$230.5 \mathrm{mg} / \mathrm{L}$. Coagulation profile revealed PT (INR) 1.49 and PTTK 32.6 seconds (control: 29.6 seconds). Routine urine examination detected red cell count of $2,142 / \mu \mathrm{L}$. The chest X-ray showed bilateral airspace consolidation with relative apical sparing (Fig. 1).

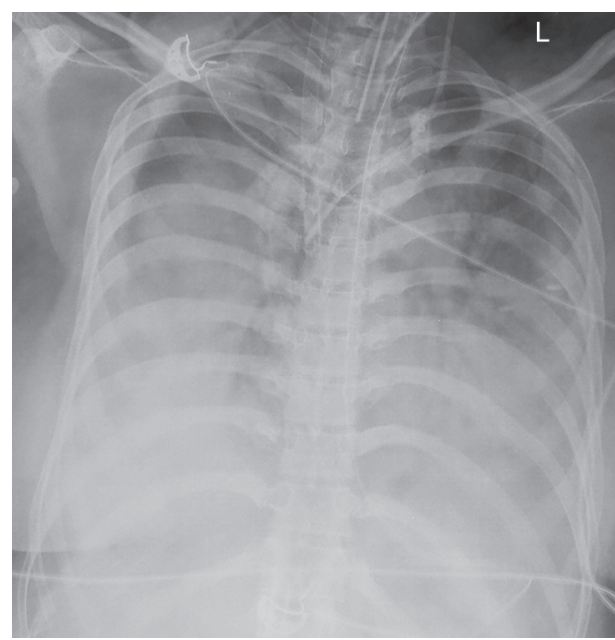

Fig. 1: Chest X-ray on day 1 showing bilateral airspace consolidation sparing the apices of lung parenchyma
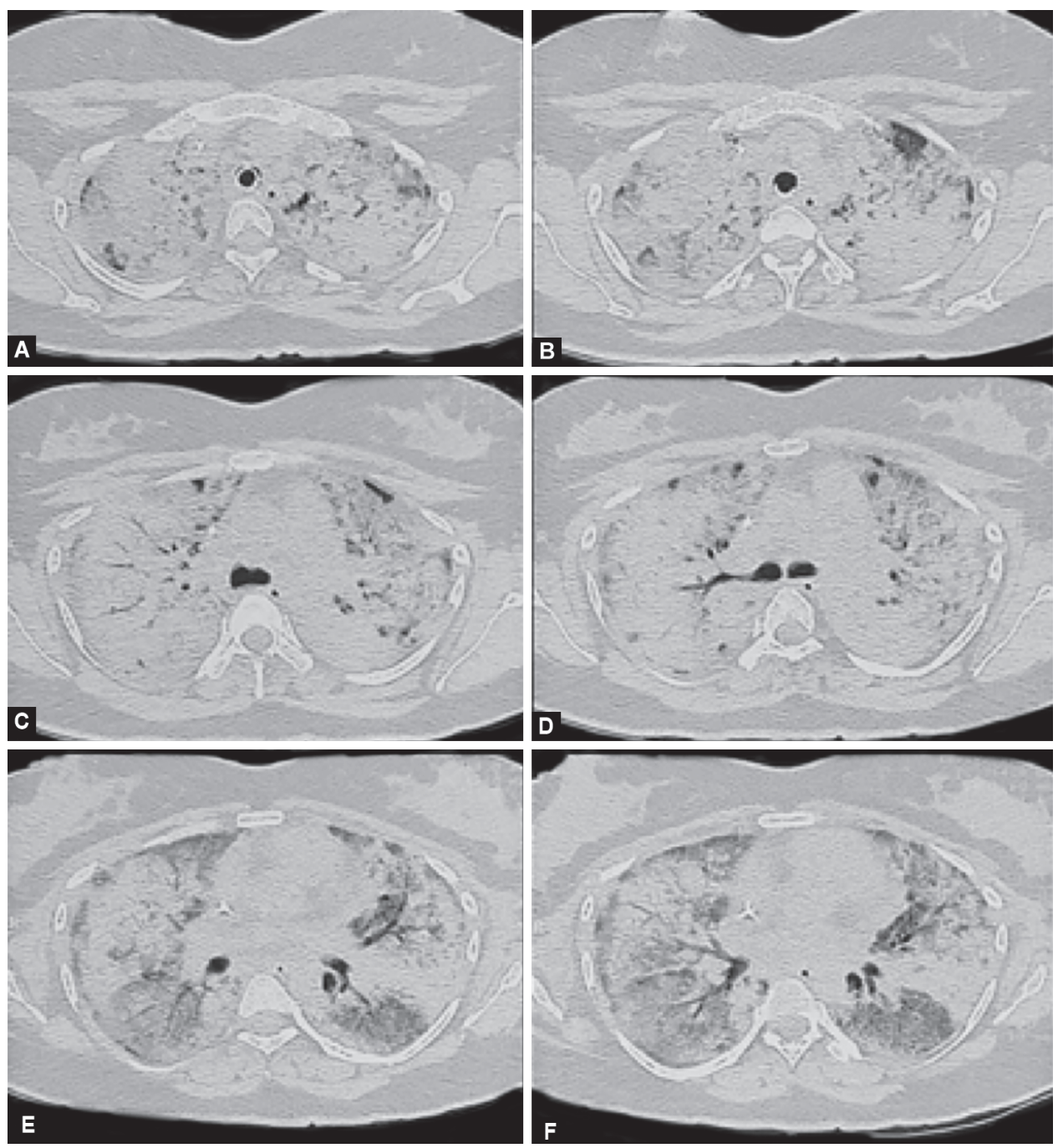

Figs 2 A to F: High-resolution computed tomography of the chest showing bilateral consolidation with diffuse alveolar filling pattern and ground glass haze

Two-dimensional ECHO findings indicated normal left ventricular ejection fraction $55 \%$ with no regional wall motion abnormalities. High-resolution computed tomography (HRCT) of the chest revealed bilateral consolidation with diffuse alveolar filling pattern and ground-glass haze (Fig. 2). Based on the clinical history of hemoptysis, acute hypoxemic respiratory failure, coupled with low hemoglobin in routine blood tests and typical HRCT chest findings, a possibility of diffuse alveolar hemorrhage was considered. A quick flexible bronchoscopy was performed through the endotracheal tube. Cytology examination of bronchoalveolar lavage fluid showed the presence of hemosiderin laden macrophages. Pulse methylprednisolone therapy was initiated immediately with a dose of $1 \mathrm{~g}$ administered intravenously. A recruitment maneuver was applied on CMV mode, but the patient continued to remain hypoxic. The mode was changed to pressure-control inverse-ratio ventilation (PCIRV) with Pmax $30 \mathrm{~cm} \mathrm{H}_{2} \mathrm{O}, \mathrm{RR} 32$ /minute, I:E ratio 1.5:1, $\mathrm{FiO}_{2} 100 \%$, and PEEP $12 \mathrm{~cm} \mathrm{H}_{2} \mathrm{O}$. ABG taken after 2 hours showed pH 7.119, $\mathrm{pCO}_{2} 67.6 \mathrm{~mm} \mathrm{Hg}, \mathrm{pO}_{2} 54.4 \mathrm{~mm} \mathrm{Hg}, \mathrm{HCO}_{3} 17.6$ $\mathrm{mmol} / \mathrm{L}, \mathrm{SpO}_{2} 77 \%$, and lactate $11.4 \mathrm{mmol} / \mathrm{L}$. Lung compliance was $18 \mathrm{~mL} / \mathrm{cm} \mathrm{H}_{2} \mathrm{O}$. After 4 hours of mechanical ventilation, due to persistence of intractable hypoxia with the $\mathrm{PaO}_{2}-\mathrm{FiO}_{2}$ ratio of 54.4 and Murray Score of 3.8 points, we decided to rescue her with veno-venous extracorporeal membrane oxygenation (V-V ECMO). 


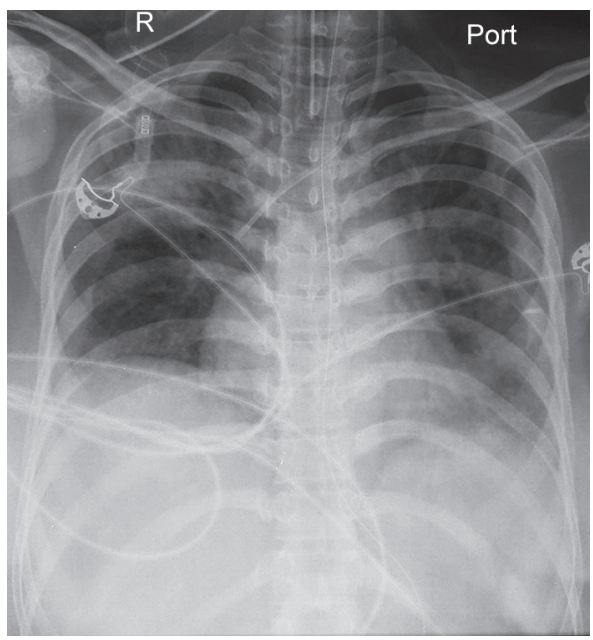

Fig. 3: Chest X-ray on day 7 showing significant resolution of the airspace opacities

Percutaneous insertion of an outflow $22 \mathrm{~F}$ venous cannula (Edwards ${ }^{\circledR}$ Lifesciences Corporation, Irvine, USA) in the right femoral vein and $20 \mathrm{~F}$ arterial cannula (Edwards ${ }^{\circledR}$ Lifesciences Corporation) for return in the right internal jugular vein was done under ultrasound guidance. V-VECMO was initiated on a Deltastream ${ }^{\circledR}$ DP3 with HILITE 7000 LT oxygenator (Medos Cardiopulmonary Solutions, Stolberg, Germany). The initial ECMO flow rate was set at $4 \mathrm{~L} / \mathrm{min}$ and sweep gas at $3 \mathrm{~L} /$ minute with $\mathrm{FiO}_{2}$ of 1.0 . The $\mathrm{SpO}_{2}$ rose quickly to over $97 \%$ after initiating ECMO. For anticoagulation, the activated clotting time (ACT) was maintained within a range of 140-160 seconds using unfractionated heparin infusion. Renal support was also initiated with continuous veno-venous hemodiafiltration (CVVHDF) due to deranged renal function and anuria. Her immunological profile results showed elevated titers of ANA 1:320, cytoplasmic ANCAs 1:160, and serine proteinase 3 antibodies (anti-PR3) $31.2 \mathrm{U}$ (negative $<3.5$ ). Hence, a final diagnosis of AAV with diffuse alveolar hemorrhage with pulmonary and renal involvement was established. The patient was treated with methylprednisolone $1 \mathrm{~g}$ daily for 3 days followed by a pulse dose of cyclophosphamide $1 \mathrm{~g}$ intravenously. Five cycles of plasmapheresis were also given. The patient responded favorably with gradual improvement in clinical, radiological (Fig. 3), and ventilator parameters in the subsequent days. The CVVHDF could be stopped on day 4, ECMO was weaned off on day 7 , and the patient was successfully extubated from the ventilator on day 9 .

\section{Discussion}

Diffuse alveolar hemorrhage is often a life-threating syndrome that may present with acute respiratory failure. The mortality is reported as $77 \%$ when mechanical ventilation is required in these cases. ${ }^{4,5}$ ECMO was initiated in this patient, as she had lifethreatening refractory hypoxemia with a calculated mortality risk of $80 \%$ according to the ESLO criteria. ${ }^{1}$ Prone position ventilation, although considered, could not be attempted before initiating ECMO due to hemodynamic instability. Successful use of ECMO has been described in patients with DAH due to vasculitis and other autoimmune disorders mainly in the form of case reports. ${ }^{2,3,6-10}$ In the largest series of 19 cases enrolled over a period of 10 years, Seelinger et al. ${ }^{11}$ described that VV-ECMO in refractory respiratory failure due to DAH was feasible, did not lead to aggravation of pulmonary bleeding, and was associated with a hospital survival rate of 53\%. However, they reported fatal intracranial hemorrhage in $16 \%$ of the cases which was possibly due to global endothelial injury and inflammation as part of the underlying pathophysiology of vasculitis and was not related to ECMO.

In this patient, lower than the usual range of ACT was targeted for achieving anticoagulation. Earlier reports ${ }^{2,3}$ observed that the use of anticoagulation during ECMO in patients with DAH may not be associated with increased bleeding risks. Cases with use of heparin-free ECMO with favorable outcome have also been reported. ${ }^{6-8}$ However, avoidance of anticoagulation can lead to circuit thrombosis and systemic thromboembolism. Novel surface material and coating in the modern ECMO oxygenators and circuits are likely to render anticoagulation unnecessary, but this approach is far from being routinely implemented. ECMO proved to be lifesaving in our patient who was initiated on prompt immunosuppressive therapy and plasmapheresis along with other supportive care involving hemodynamic management and CVVHD. We feel that ECMO could be considered as adjunctive therapy in severe hypoxemic respiratory failure associated with DAH after careful consideration of the risk of bleeding and a restrictive anticoagulation strategy.

\section{References}

1. ELSO Adult Respiratory Failure Supplement to the ELSO General Guidelines. Version 1.4 August 2017.

2. Rawal G, Kumar R, Yadav S. ECMO rescue therapy in diffuse alveolar haemorrhage: a case report with review of literature. J Clin Diagn Res 2016;10(6):OD10-OD11. DOI: 10.7860/JCDR/2016/20649.7969.

3. Delvino P, Monti S, Balduzzi S, Belliato M, Montecucco C, Caporali $\mathrm{R}$. The role of extra-corporeal membrane oxygenation (ECMO) in the treatment of diffuse alveolar haemorrhage secondary to ANCAassociated vasculitis: report of two cases and review of the literature. Rheumatol Int 2019;39(2):367-375. DOI: 10.1007/s00296-018-4116-z.

4. West S, Arulkumaran N, Ind PW, Pusey CD. Diffuse alveolar haemorrhage in ANCA-associated vasculitis. Intern Med 2013;52(1):513. DOI: 10.2169/internalmedicine.52.8863.

5. Quadrelli S, Dubinsky D, Solis M, Yucra D, Hernández M, Karlen H, et al. Immune diffuse alveolar hemorrhage. Clinical presentation and outcome. Respir Med 2017;129:59-62.

6. Patel JJ, Lipchik RJ. Systemic lupus-induced diffuse alveolar haemorrhage treated with extracorporeal membrane oxygenation: a case report and review of the literature. J Intensive Care Med 2014;29(2):104-109. DOI: 10.1177/0885066612464335.

7. Hohenforst-Schmidt W, Petermann A, Visouli A, Zarogoulidis P, Darwiche K, Kougioumtzi I, et al. Successful application of extracorporeal membrane oxygenation due to pulmonary haemorrhage secondary to granulomatosis with polyangiitis. Drug Des Devel Ther 2013;7:627-633. DOI: 10.2147/DDDT.S47156.

8. Claudio CP, Charbonney E, Durand M, Kolan C, Laskine M. Extracorporeal membrane oxygenation in diffuse alveolar haemorrhage secondary to systemic lupus erythematosus. J Clin Med Res 2014;6:145-148.

9. Zhen G, Xin L, Li-ya J, Ling-feng X. Extracorporeal membrane oxygenation for the management of respiratory failure caused by diffuse alveolar haemorrhage. J Extra Corpor Technol 2009;41:37-40.

10. Barnes SL, Naughton M, Douglass J, Murphy D. Extracorporeal membrane oxygenation with plasma exchange in a patient with alveolar haemorrhage secondary to Wegener's granulomatosis. Intern Med J 2012;42(3):341-342. DOI: 10.1111/j.1445-5994.2012.02720.x.

11. Seeliger B, Stahl K, Schenk H, Schmidt JJ, Wiesner O, Welte T, et al. Extracorporeal membrane oxygenation for severe ARDS due to immune diffuse alveolar hemorrhage. A retrospective observational study. Chest 2020;157(3):744-777. DOI: 10.1016/j.chest.2019. 10.021 . 